\title{
Sudden cardiac arrest in a young adult with long QT syndrome and negative mutation studies
}

\author{
Walter Duarte MD, Hawa Edriss MD
}

\begin{abstract}
Cardiac arrest in young healthy adults usually results from undiagnosed structural heart disease or an undiagnosed predisposition to cardiac arrhythmias. We report a 19-year-old man who developed a cardiac arrest while playing golf. He required cardiopulmonary resuscitation on 5 separate occasions and then was treated with targeted temperature management. His initial laboratory tests included hypokalemia $(2.5 \mathrm{mmol} / \mathrm{L})$; he also had several episodes of hypoglycemia during his intensive care unit stay. A serum insulin level was within normal limits, but a C-peptide level was increased. He had a long QT interval (QTc-551 ms) after rewarming. Extensive cardiac workup was negative for structural abnormalities. In addition, mutation testing for channelopathies was negative. This patient recovered and has done well. This patient illustrates a case of sudden cardiac death in a young healthy adult who had a long QT syndrome but a negative mutation analysis. These patients might benefit from evaluation in specialized centers which can undertake additional genetic testing.
\end{abstract}

Keywords: channelopathies, long QT syndrome, sudden death, reactive hypoglycemia

\section{INTRODUCTION}

Long QT syndrome (LQTS) is a cardiac electrophysiological disorder characterized by a delayed cardiac repolarization, prolonged QT intervals on ECG, syncope, malignant arrhythmias, and sudden death. ${ }^{1}$ There are 15 genes known to be associated with LQTS, of which the potassium voltage-gated channel subfamily $Q$ member 1 (KCNQ1), the potassium voltage-gated channel subfamily $\mathrm{H}$ member $2(\mathrm{KCNH} 2)$, and the sodium channel protein type 5 alpha unit (SCN5A) are the most common. ${ }^{2} \mathrm{KCNQ} 1$ and $\mathrm{KCNH} 2$ genes encode a voltage-gated $\mathrm{K}+$ channel located in both cardiomyocytes and pancreatic beta-cells. Loss of function or mutation in the KCNQ1 or $\mathrm{KCNH} 2$ genes

Corresponding author: Walter Duarte

Contact Information: Wr.duarte-celada@ttuhsc.edu DOI: 10.12746/swrccc.v7i27.520 causes long QT syndrome (LQTS) and increases in insulin secretion. ${ }^{3,4}$

The phenotype of patients with LQTS, caused by mutation in KCNQ1 and $\mathrm{KCNH} 2$, includes prolonged cardiac repolarization, hyperinsulinemia, and hypoglycemia. Patients with KCNQ1 mutations can also present with low potassium levels. LQTS secondary to inherited mutations affects up to 1:2000 people.,4 Twenty percent of the patients who meet the clinical diagnostic criteria for LQTS do not have detectable pathogenic variants in one or more of the 15 genes known to be associated with LQTS. ${ }^{2}$

\section{CASE}

A 19-year-old male patient with no significant past medical history presented to the Emergency Department (ED) after a sudden cardiac arrest. On the day of admission, the patient was playing golf when he suddenly collapsed, became unresponsive, 
and lost his pulse. When the emergency medical service arrived, he was gasping, pale, and pulseless. Cardiopulmonary resuscitation (CPR) was started. The initial rhythm was ventricular fibrillation (VF), and he then developed pulseless electrical activity (PEA). After 2 rounds of CPR he regained spontaneous circulation and was intubated. Then he had a cardiac arrest again, and after 2 more rounds of $\mathrm{CPR}$, he regained spontaneous circulation. Prior similar episodes, use of recreational drugs, and a family history of cardiac problems were denied by his parents.

On his arrival to the ED at University Medical Center, the patient had a cardiac arrest for the 3rd time. He regained circulation after one round of CPR. On physical examination the patient was unresponsive, intubated, afebrile, hypertensive $(160 / 90 \mathrm{mmHg})$, and tachycardic (120 bmp). His cardiopulmonary examination was unremarkable, and his skin was pale and free of bruises or lesions.

Laboratory studies revealed white blood cell count $11.5 \times 10^{3} / \mathrm{mm}^{3}$, hemoglobin $13.5 \mathrm{gm} / \mathrm{dL}$, creatinine $1.0 \mathrm{mg} / \mathrm{dL}$, glucose $306 \mathrm{mg} / \mathrm{dL}$, sodium $147 \mathrm{mmol} / \mathrm{L}$, potassium $2.5 \mathrm{mmol} / \mathrm{L}$, and calcium $6.8 \mathrm{mg} / \mathrm{dL}$. Troponins were elevated $(0.05 \mathrm{ng} / \mathrm{mL})$. Lactate was $9.8 \mathrm{mmol} / \mathrm{L}$. His electrocardiogram (ECG) showed a right bundle branch block (RBBB). The chest x-ray showed pulmonary edema. A urine drug screen was negative. An echocardiogram at the bedside showed global hypokinesis, a dilated left ventricle, and a right ventricle with normal size and function. The patient was admitted to the Medical Intensive Care Unit (MICU) and started on a targeted temperature management protocol for 48 hours. Computed tomography scans of the head, chest, abdomen, and pelvis were unremarkable. Thyroid stimulating hormone was $4.57 \mathrm{mIU} / \mathrm{mL}$, and cortisol was $12 \mathrm{mcg} / \mathrm{dL}$. Blood, respiratory, and urine cultures were negative.

After targeted temperature management was completed, Brugada syndrome was ruled out. A repeat ECG (24 hours post therapeutic hypothermia) showed incomplete RBBB, T wave inversion in antero-lateral leads, and QT prolongation (QTc: $551 \mathrm{~ms}$ ). The patient was started on metoprolol. Forty-eight hours post cardiac arrest, a transesophageal echocardiogram was performed and reported an ejection fraction of $55-59 \%$ and normal left ventricular motion.

During the course of this hospitalization, the patient had several episodes of hypoglycemia, ranging from 44-63 mg/dL (Table). He also had hypokalemia on admission $(2.5 \mathrm{mmol} / \mathrm{L})$ and at several other times with values between 3.2-3.4 mmol/L (Table). His insulin level and C-peptide level were $3.8 \mathrm{mU} / \mathrm{mL}$ (normal) and $4.45 \mathrm{ng} / \mathrm{mL}$ (elevated), respectively. During hospitalization, the patient developed rhabdomyolysis which resolved with intravenous fluids. Cardiac magnetic resonance imaging was unremarkable. The patient improved, was extubated on day 4 after admission, and was transferred out of the MICU on day 6. An implantable cardio-defibrillator (ICD) was placed by cardiology as a secondary prevention for sudden cardiac death. His clinical course continued without complications, and the patient was discharged on day 9. Patient was reevaluated 1 month after discharge and was doing well. Genetic testing for channelopathies was requested, and no pathogenic variant was detected.

\section{Discussion}

We present a case of a young male patient with no past medical history who had three cardiac arrests. The ECG after 48 hours of targeted temperature management showed a prolonged QT interval (QTC: 551$)$. With this presentation the patient met the Schwartz clinical criteria for high probability of LQTS. ${ }^{5}$ During the hospitalization, he was studied extensively for the main causes of cardiac arrest in this age group. Drug intoxication, cardiac structural abnormalities, and Brugada syndrome were ruled out. The other possibility was a channelopathy as a cause of this cardiac arrest and QT prolongation, but all genetic variants tested were negative. The genetic testing was also negative for mutations related with catecholaminergic polymorphic ventricular tachycardia, and the cardiac MRI ruled out structural abnormalities, such as arrhythmogenic right ventricular cardiomyopathy/ dysplasia, which are less frequent but known causes of arrhythmias in young patients.

Sudden unexplained death/cardiac arrest in apparently healthy individuals with no definite cause after 
Table.

\begin{tabular}{|c|c|c|c|c|c|}
\hline Hospital Day & Day 1 & Day 2 & Day 3 & Day 4 & Day 5 \\
\hline Progress & $\begin{array}{l}\text { Admitted to } \\
\text { MICU 13:00 }\end{array}$ & & & Extubated & \\
\hline Temperature & $\begin{array}{l}\text { Hypothermia } \\
\text { started at } 16: 50 \\
20: 45-96^{\circ} \mathrm{F} \\
21: 15-94^{\circ} \mathrm{F} \\
22: 15-92^{\circ} \mathrm{F}\end{array}$ & $90^{\circ} \mathrm{F}$ & $\begin{array}{l}\text { Rewarming } \\
\text { started at } 6: 00 \\
\text { to } 7: 00 \text { am } \\
7: 00-95.0^{\circ} \mathrm{F} \\
10: 00-96.0^{\circ} \mathrm{F} \\
17: 00-97.8^{\circ} \mathrm{F} \\
\text { Rewarming } \\
\text { Completed }\end{array}$ & & \\
\hline $\begin{array}{l}\text { Glucose } \\
\text { mg/dL }\end{array}$ & 306 & & $\begin{array}{l}00: 08-56 \\
03: 07-56 \\
07: 15-44 \\
17: 11-63\end{array}$ & & \\
\hline $\mathrm{K} \mathrm{mmol} / \mathrm{L}$ & 2.5 & 4.1 & 3.7 & 3.2 & 3.4 \\
\hline $\begin{array}{l}\text { C peptide } \\
\text { ng/mL }\end{array}$ & & & $\begin{array}{l}4.4 \text { (elevated) } \\
18: 37\end{array}$ & & \\
\hline $\begin{array}{l}\text { Insulin } \\
\mathrm{mU} / \mathrm{mL}\end{array}$ & & & $\begin{array}{l}3.8 \text { (normal) } \\
18: 37\end{array}$ & & \\
\hline
\end{tabular}

comprehensive investigation presents a challenge to physicians. Cardiac channelopathies, a group of inheritable diseases that primarily affect heart rhythm by altering the cardiac conduction, have been known as one of the more likely causes of cardiac arrest/sudden death. The major channelopathy genes related to LQTS are the potassium voltage-gated channel subfamily $\mathrm{Q}$ member 1 (KCNQ1), the potassium voltage-gated channel subfamily $\mathrm{H}$ member $2(\mathrm{KCNH} 2)$, the sodium channel protein type 5 alpha unit (SCN5A), and potassium voltage-gated channel protein Isk-related family member 1 (KCNE1) and 2 (KCNE2). ${ }^{6}$ The most prevalent LQTS variant is caused by the mutations in the KCNQ1 gene, and approximately half of genotyped patients have this mutation. The next most common causes are $\mathrm{KCNH} 2$ and $\mathrm{SCN} 5 \mathrm{~A}$ mutations which range from $35-40 \%$ and $10-15 \%$, respectively. ${ }^{4,7}$ It is estimated that $10-35 \%$ of sudden unexplained death are explained by cardiac channelopathies. ${ }^{6}$

Cardiac channelopathies associated with sudden death have been reported in all ethnic groups, with an African Americans predominance. ${ }^{6}$ According to the Centers for Disease Control and Prevention, more than 4500 infants die suddenly each year in the United States (U.S.) with no obvious cause of death; half of those deaths remains unexplained after investigation. The prevalence of sudden unexplained deaths in patients between 1-22 years exceeds 2000 annually in the U.S. ${ }^{8}$ Most channelopathies are inherited in an autosomal dominant manner but have reduced penetrance and variable expression. ${ }^{6}$

This patient also had episodes of hypoglycemia and hypokalemia which suggested the diagnosis of hyperinsulinemia or reactive hypoglycemia. The insulin level was measured 12 hours after rewarming and was within the normal range, but the C-peptide level was elevated. Hypothermia and the rewarming process can cause metabolic abnormalities, including glycemic excursions, mostly hyperglycemia, insulin resistance, ${ }^{9,10}$ and hypokalemia. ${ }^{11,12}$ Our patient presented with hypokalemia $(2.5 \mathrm{mmol} / \mathrm{L})$ on admission before the hypothermia protocol and had more 
episodes of hypokalemia 12 and 36 hours after normothermia was reached after hypothermia. He also had episodes of hypoglycemia during the hypothermia protocol, prior to rewarming phase, which is associated with increased glucose variability.

Cardiac arrest initiates a cascade of metabolic and electrolyte disturbances likely secondary to shifts from aerobic to anaerobic metabolism. Cardiac arrest can cause hypokalemia due to rapid reversal of acidemia with bicarbonate solutions and hyperventilation or due to beta agonists (adrenaline) leading to potassium shifts. ${ }^{13}$ Although patients can develop hypokalemia after arrest, our patient presented with hypokalemia and continued to have episodes of low potassium even after rewarming and extubation. We think that hypokalemia was part of the cause and not the consequence of the cardiac arrest. In addition, therapeutic hypothermia can cause electrocardiographic changes, including QT prolongation, ${ }^{14,15}$ which is usually reversible after the rewarming phase has been completed. For that reason, the ECG on our patient was done 24 hours after the normothermia was reached and showed a markedly prolonged QT (QTc:551 ms).

The combination of cardiac arrest and hypoglycemia could be explained by a gene mutation in this patient. On admission, KCNQ1 or $\mathrm{KCNH} 2$ gene mutations were considered the cause of his clinical presentation. KCNQ1 encodes a voltage-gated $\mathrm{K}+$ channel, located in both cardiomyocytes and pancreatic beta cells. $\mathrm{KCNH} 2$ is present in cardiomyocytes, pancreatic alpha and beta cells, and intestinal $L$ and $\mathrm{K}$ cells. Both these mutations are characterized by a delayed repolarization. Loss of function or mutation in $\mathrm{KCNQ} 1$ and $\mathrm{KCNH} 2$ cause long QT syndrome (LQTS) and increased insulin secretion. ${ }^{3,4}$ Although no pathological variant was found on genetic testing of our patient, there might be other mutations that predispose to this type of pathology and not yet discovered. Forty-six genes related to cardiac arrhythmias were tested in this case, and all were negative.

Hyltén-Cavallius et al reported that patients with LQTS secondary to $\mathrm{KCNH} 2$ mutation had increased serum insulin, serum C-peptide, and decreased plasma glucose levels after glucose ingestion (reactive hypoglycemia) compared to matched control patients. Defective glucagon secretion was also associated with this mutation. A higher proportion of patients with $\mathrm{KCNH} 2$ mutation developed hypoglycemia $(63 \%)$ compared to the control group (36\%). In this study no differences in potassium response were reported. ${ }^{4}$ After glucose ingestion, more adenosine triphosphate (ATP) is produced, which closes K-ATP channels. This may prolong the repolarization phase and thus prolong the QT interval in cardiomyocytes.

Although some patients can have symptoms at rest, the triggers for most of the arrhythmias of LQTS are stress related, secondary to sudden increases in sympathetic activity. For this reason, the first line of treatment are the antiadrenergic medications, such as beta-blocker drugs. It is well known that the response to beta blockers depends on the genotype with better responses in patients with KCNQ1. Other options of treatment are the left cardiac sympathetic denervation or implantable cardioverter defibrillators (ICDs), which can be considered depending on the clinical presentation of the disease and response to beta-blockers. In patients who had a cardiac arrest, an ICD should be placed immediately. ${ }^{7}$

Molecular screening is now part of the diagnosis process of LQTS. And the identification of the genetic defect is also important for the family, serving as a screening tool for the rest of family members who could be at risk. ${ }^{7}$ It is important to mention that approximately $20 \%$ of patients meeting the clinical diagnostic criteria for LQTS do not have detectable pathogenic variants in one or more of the 15 genes known to be associated with LQTS. ${ }^{2}$ The clinical relevance of the gene mutations is based on the American College of Medical Genetics recommendations, where some variations are recognized as a direct cause of the disorder, and others may or may not be the cause of the disorder. ${ }^{16}$

\section{Conclusions}

As molecular science continues to develop more sensitive tools, it is important that physicians be aware of LQTS mutation negative cases and of the phenotypic variations, including combinations of symptoms, severity, and metabolic disturbances to identify more genes involved in prolonged QT intervals. 
Article citation: Duarte W, Edriss H. Sudden cardiac arrest in a young adult with long QT syndrome and negative mutation studies. The Southwest Respiratory and Critical Care Chronicles 2019;7(27):67-71

From: The Departments of Neurology (WD) and Internal Medicine (HE) at Texas Tech University Health Sciences Center in Lubbock, Texas

Submitted: $11 / 12 / 2018$

Accepted: 12/23/2018

Reviewer: Pooja Sethi MD, Olusegun Oyenuga MD

Conflicts of interest: none

This work is licensed under a Creative Commons

Attribution-ShareAlike 4.0 International License

\section{REFERENCES}

1. Moss AJ, Shimizu W, Wilde AA, et al. Clinical aspects of type 1 long QT syndrome by location, coding type, and biophysical function of mutations involving the KCNQ1 gene. Circulation 2007;115:2481-2489.

2. Alders M, Bikker H, Christians I. Long QT syndrome. Gene Reviews. Seattle (WA): University of Washington, Seattle; 1993-2018.

3. Torekov SS, Iepsen E, Christiansen M, et al. KCNQ1 long QT syndrome patients have hyperinsulinemia and symptomatic hypoglycemia. Diabetes 2014;63:1315-25.

4. Hyltén-Cavallius L, Iepsen EW, Wewer Albrechtsen NJ, et al. Patients with Long-QT syndrome caused by impaired hERG-encoded Kv 11.1 potassium channel have exaggerated endocrine pancreatic and incretin function associated with reactive hypoglycemia. Circulation 2017;135:1705-19.

5. Schwartz PJ. The congenital long QT syndromes from genotype to phenotype: clinical implications. J Intern Med 2006; 259:39-47.
6. Wang D, Shah KR, Um SY, et al. Cardiac channelopathy testing in 274 ethnically diverse sudden unexplained deaths. Forensic Sci Int 2014;237:90-9.

7. Crotti L, Celano G, Dagradi F, et al. Congenital long QT syndrome. Orphanet J Rare Dis 2008;3:18.

8. Ackerman MJ, Tester DJ, Driscoll DJ. Molecular autopsy of sudden unexplained death in the young. Am J Forensic Med Pathol 2001;22:105-11.

9. Cueni-Villoz N, Devigili A, Delodder F, et al. Increased blood glucose variability during therapeutic hypothermia and outcome after cardiac arrest. Crit Care Med 2011;39:2225-31.

10. Haase KK, Grelle JL, Khasawneh FA, et al. Variability in glycemic control with temperature transitions during therapeutic hypothermia. Crit Care Res Pract 2017;2017:4831480.

11. Soeholm H, Kirkegaard H. Serum potassium changes during therapeutic hypothermia after out-of-hospital cardiac arrestshould it be treated? Ther Hypothermia Temp Manag 2012; 2:30-6.

12. Mirzoyev SA, McLeod CJ, Bunch TJ, et al. Hypokalemia during cooling phase of therapeutic hypothermia and its impact on arrhythmogenesis. Resuscitation 2010;81:1632-6.

13. Bellomo R, Martensson J, Eastwood GM. Metabolic and electrolyte disturbances after cardiac arrest: How to deal with it. Best Pract Res Clin Anaesthesiol 2015;29:471-84.

14. Kim SM, Hwang GS, Park JS, et al. The pattern of TpeakTend and QT interval, and J wave during therapeutic hypothermia. J Electrocardiol 2014;47:84-92.

15. Salinas P, Lopez-de-Sa E, Pena-Conde L, et al. Electrocardiographic changes during induced therapuetic hypothermia in comatose survivors after cardiac arrest. World J Cardiol 2015; 7:423-30.

16. Richards CS, Bale S, Bellissimo DB, et al. ACMG recommendations for standards for interpretation and reporting of sequence variations: revision 2007. Genet Med 2008;10: 294-300. 